\title{
META-IMAGE - A COLLABORATIVE ENVIRONMENT FOR IMAGE DISCOURSE
}

\author{
Lisa Dieckmann ${ }^{1)}$, Anita Kliemann ${ }^{2)}$, Martin Warnke ${ }^{3)}$ \\ 1) prometheus - Das verteilte digitale Bildarchiv für Forschung \& Lehre Kunsthistorisches Institut der Universität zu \\ Köln Albertus-Magnus-Platz, D-50923 Köln, lisa.dieckmann@uni-koeln.de, http://www.prometheus-bildarchiv.de \\ 2) Scharnhorststraße 1, D-21337 Lüneburg, kliemann@leuphana.de, http://www.meta-image.de \\ 3) Scharnhorststraße 1, D-21337 Lüneburg, warnke@leuphana.de, http://www.meta-image.de
}

\begin{abstract}
The aim of the project Meta-Image, funded by German Research Foundation (DFG), is to provide a networkbased research environment for art history and other sciences concerning visual culture. It consists of the two components prometheus and HyperImage. Meta-Image combines the distributed digital image archive prometheus, which consists of a very large pool of images, with HyperImage (http://www.hyperimage.eu), a tool for image annotation. prometheus provides over 700,000 images in nearly 60 connected image databases; Hyperimage facilitates collaborative work directly on the image. The numerous users, the secure legal context for use and the existing technologies for collaborative research make prometheus a perfect subject for HyperImage. This image annotation tool serves as an instrument to support research in art history. It allows the identification of motifs, the creation of linked image networks as well as the addition of metadata. This synthesis creates the ability to reorganise, juxtapose and annotate images in a way that can lead to new conclusions concerning image-based research. Art history and other cultural studies can finally realise the potential of the network based and collaborative analysis of images.
\end{abstract}

Keywords: collaborative research, visual culture, art history, image-based research, network-based research, image database, Aby Warburg, linking, annotations.

\section{INTRODUCTION}

The aim of the DFG-project Meta-Image (http://www.meta-image.de) is to provide a networkbased research environment for art history and other sciences concerning visual culture, that enables collaborative research directly on images.

One of the preconditions for DFG funding was to develop a new research environment by using existing technologies. Meta-Image attempts this: it combines the distributed digital image archive prometheus (http://www.prometheus-bildarchiv.de), consisting of a very large pool of images, with HyperImage (http://www.hyperimage.eu), a tool for image annotation. The components of both are combined in Meta-Image. The key benefit to image discourse will be network-based collaborative research.

The following section introduces the fields of discourse which form the theoretical background of the project Meta-Image. It refers above all to the iconographic and iconological work of the art historian and cultural theorist Aby Warburg. His approach was to depict references between images and to annotate them. These techniques are essential to describing how Meta-Image works: directly on the image, using the image as a visual argument.

Meta-Image is a contemporary way for researchers to work on images, as did Warburg. Contemporary in this sense means connected, collaborative and using digital communication. Therefore Meta-Image, consisting of the networkbased applications prometheus and HyperImage and their technologies, offers what is needed to work in a Warburgian way. prometheus provides the objects for research, i.e. images while HyperImage is the tool that can be used to define visual structures and connections between images to support scientific reasoning.

In sections 3 and 4 these components of MetaImage are described in detail. How do they work? What are their main functions? What are the advantages that make them indispensable for collaborative image research?

Finally in chapter 5 the text will focus on how prometheus and HyperImage are connected to each other, how they work together and form MetaImage. The technical details concerning the connection of both parts are given as well as a description of what users are able to do with MetaImage and what the practical benefit for 
collaborative image discourse can be.

\section{IN FAVOUR OF AN IMAGE-BASED DISCOURSE}

The development of these new search and annotation technologies invoked some fundamental issues regarding the nature of images identified in post-modern philosophical discourse.

In 1994 Gottfried Boehm [1] introduced the term iconic turn into the debate about the nature of the image, alluding to the linguistic turn of Richard Rorty [2]. However, he missed the analytical approach to the image that linguistics had already proposed for text. The 'lack of meaning of an isolated linguistic element of articulation that de Saussure postulated' could also be applied to the image. A single colour spot in paintings by Césanne, Monet, Seurat and others does not 'mean' anything. It generates meaning by 'cooperation with other spots in a lateral way.' (Boehm, 1994, p. 22, transl. MW).

Thus images and pictures are not homogeneous units; they constitute an 'iconic difference' that emerges from the juxtaposition of the whole image and its details. 'What appears to us as image stems from a single basic contrast, the one between an overseeable total area and whatever is comprised within it as internal events' (Boehm, 1994, pp.29f.). So we are dealing with a sharp-eyed gaze, the identification and localisation of details within an image as a whole.

This contrasts with printed text, where there are long established reference schemas: chapters, pages, lines and characters. Scholarly debate is founded on the ability to identify, annotate and comment on a specific text by using footnotes and concordances, cross references and indexes. On the computer, hypertext mark-up offers a similarly precise addressing schema.

But images are by their nature analogue. Nelson Goodman writes of the image: 'no mark may be isolated as a unique, distinctive character (like a letter in an alphabet)' [3]. As deeply as we may delve into an image, we do not find specific elements that can be addressed as a word or a letter could be. One may think of Robert Campin's Portrait of a lady, that even made it to the front cover of a big German paper, together with its Man in the red stone, as an art historic sensation (Frankfurter Allgemeine Zeitung, September 3rd 2008; Figs 1 \& 2). Zooming in on the painting the detail of the red stone revealed the probable identity of the painter.

Like a letter or a word, an image detail, which in isolation is without meaning, can become a link to a line of arguments or a node in a network of references. Aby Warburg realised this in a physical form. The constantly re-arranged Mnemosyne Atlas was his medium. Cross-references were made by using woollen threads attached to pins on photographs. In the end, the network is constituted of references and cross-references that can make up its meaning. To quote Nelson Goodman, the meaning of an image 'depends rather on its relation with all the other marks in a dense, continuous field' (Nelson quoted in Mitchell, 1986, p. 67).

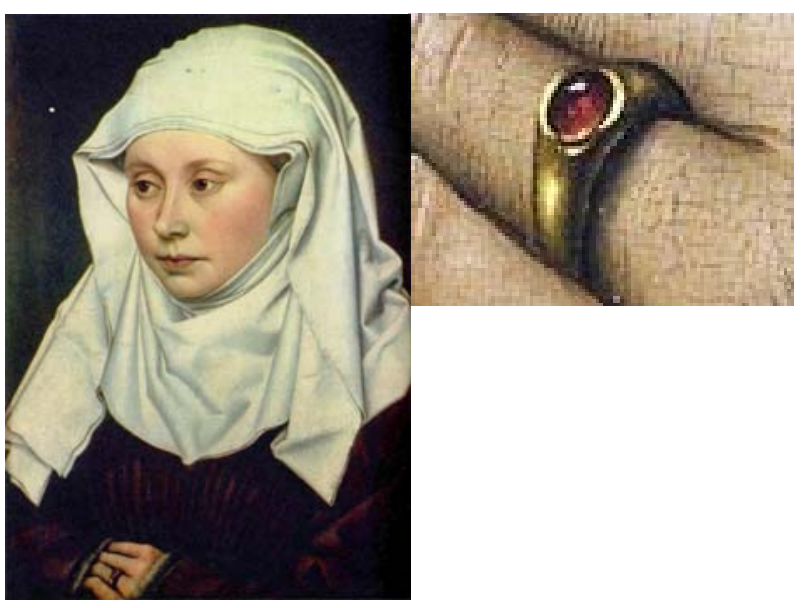

Fig. 1 and 2 - Robert Campin. Portrait of a lady 142529

This is the exact purpose of our project MetaImage. Based on the image corpus of prometheus, the distributed image archive for research and education, users can identify and link motifs by using a well-established technology (HyperImage). Analogous to hypertext links, Meta-Image allows structures and links to be identified and defined for image.

\section{PROMETHEUS - THE DISTRIBUTED IMAGE ARCHIVE FOR RESEARCH AND EDUCATION}

The initial idea of prometheus was to improve conditions for research and education in art history, archaeology and other sciences involving visual culture. Access to images as the main medium of discourse is the essential need in these subjects. When prometheus commenced in 2001 the world of humanities was still an analogue one, meaning that each individual institute maintained a slide-archive with almost the same pool of slides of canonical works of art. The advance of technology offered the opportunity to connect digital images from different sources in a virtual place, so that it was no longer necessary to hold several reproductions in many different physical locations.

After the slide archives at the various institutions had been converted into digital image archives, 
prometheus connected these databases to make the images accessible. Finally, a single search of the heterogeneous and topographically distributed, but interconnected databases replaced the individual search confined to one stand-alone slide archive. The advantages are obvious: on the one hand resources can be pooled so that just one reproduction of a work of art is needed; on the other hand an infinite number of copies can be made of a single digital image and edited without loss of quality. Because of their material independence, digital images are not liable to mechanical abrasion and chemical aging like slides and other material carriers are. Furthermore, the interconnection of the different databases breaks down canonical and disciplinary borders and widens the field of research.

From 2001 to 2005 the German Federal Ministry of education and four participating German universities funded prometheus. In 2004 the formation of a non-profit association 'prometheus the distributed digital image archive for research and education e.V' guaranteed the long-term existence and further development of the project. The financing relied on a combination of third-party funds and license fees. Since 2008 prometheus has been self-financing.

By now prometheus has connected 59 digital image archives and databases belonging to university institutes, research facilities, museums and other institutions. Altogether there are over 720,000 high-resolution images integrated as a location independent-whole (as of April 2010).

Usage rights for artists who died less than 70 years ago are regulated by Verwertungsgesellschaft Bild-Kunst (VG Bild-Kunst) in Bonn, Germany. prometheus has concluded an agreement with VG Bild-Kunst that permits making the images available through the various image databases in prometheus, and the use of those images for scientific research and educational purposes.

The technical base of prometheus is the web application framework Ruby on Rails with the search engine Ferret. Basic and advanced search functions with various possibilities for combinational searches are implemented, as well as different sorting and structuring facilities for arranging the results list.

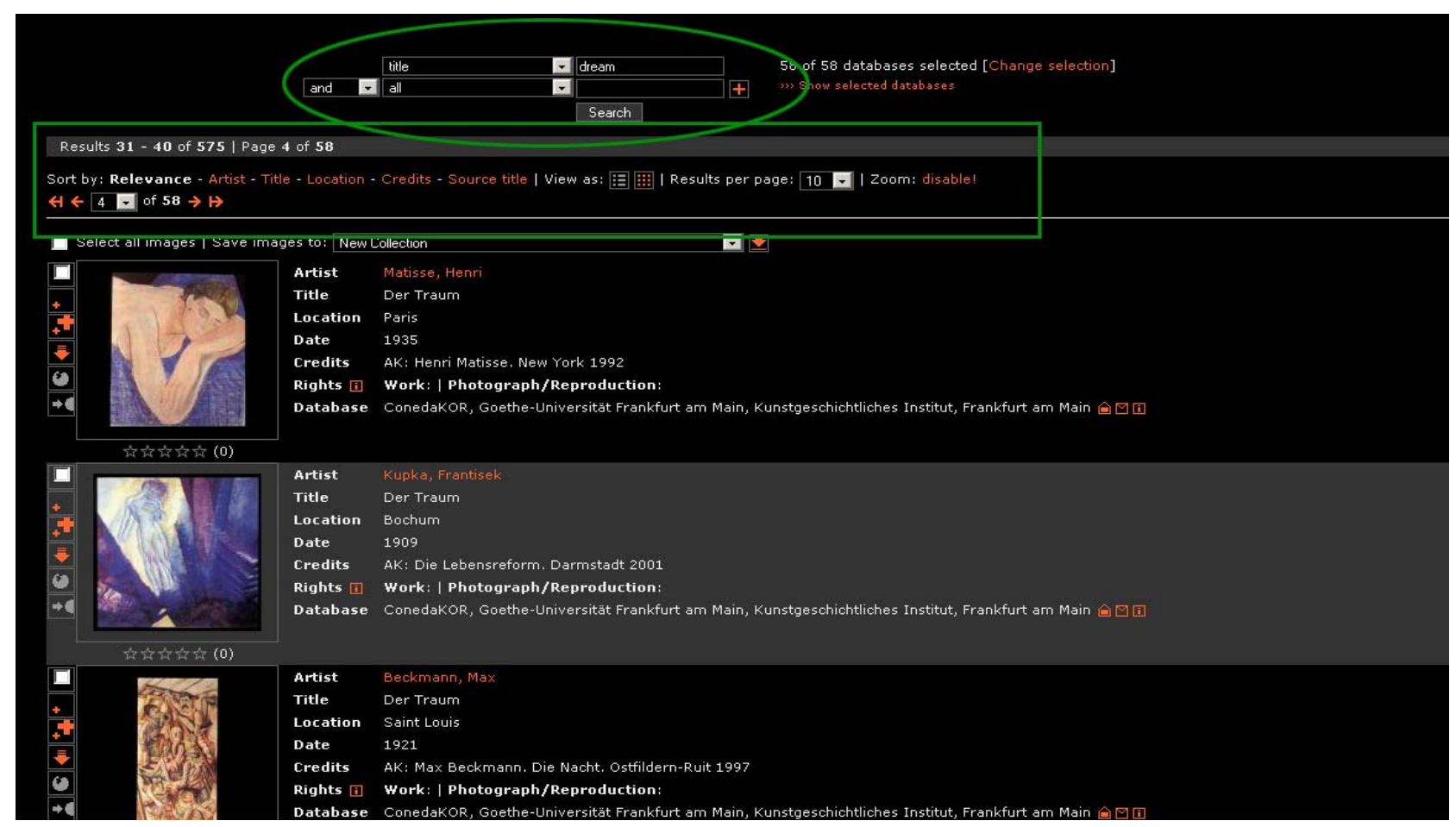

Fig. 3 - List of results in the prometheus image archive with search fields and sorting and structuring facilities

The list of results displays a thumbnail of the image and seven standard fields for every record. The full record view displays all of the data, which is held in the respective database.
As well as the medium-sized image of the related record, similar images concerning the content of a record are displayed at the bottom of the page. 


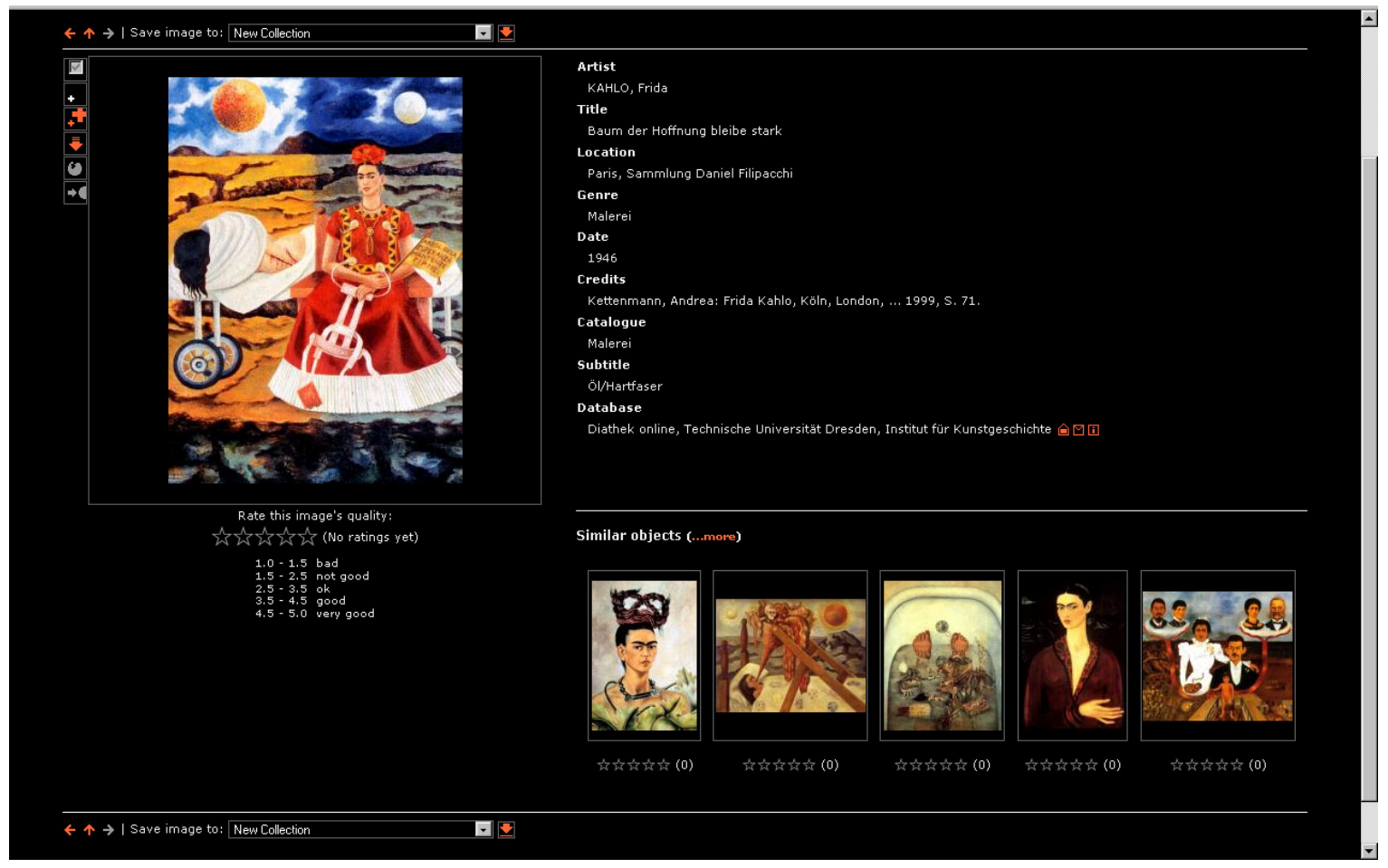

Fig. 4 - Full record view with display of similar images

A further click on the image displays the largesize high-resolution image, which has at least 1600 pixels.

Additionally, prometheus makes it possible to compile a personal collection of images for a specific topic and to add metadata to this collection.
These collections can be created for private use, or can be unlocked for public or individual users for read or write access. This enables collaborative work on the collection.

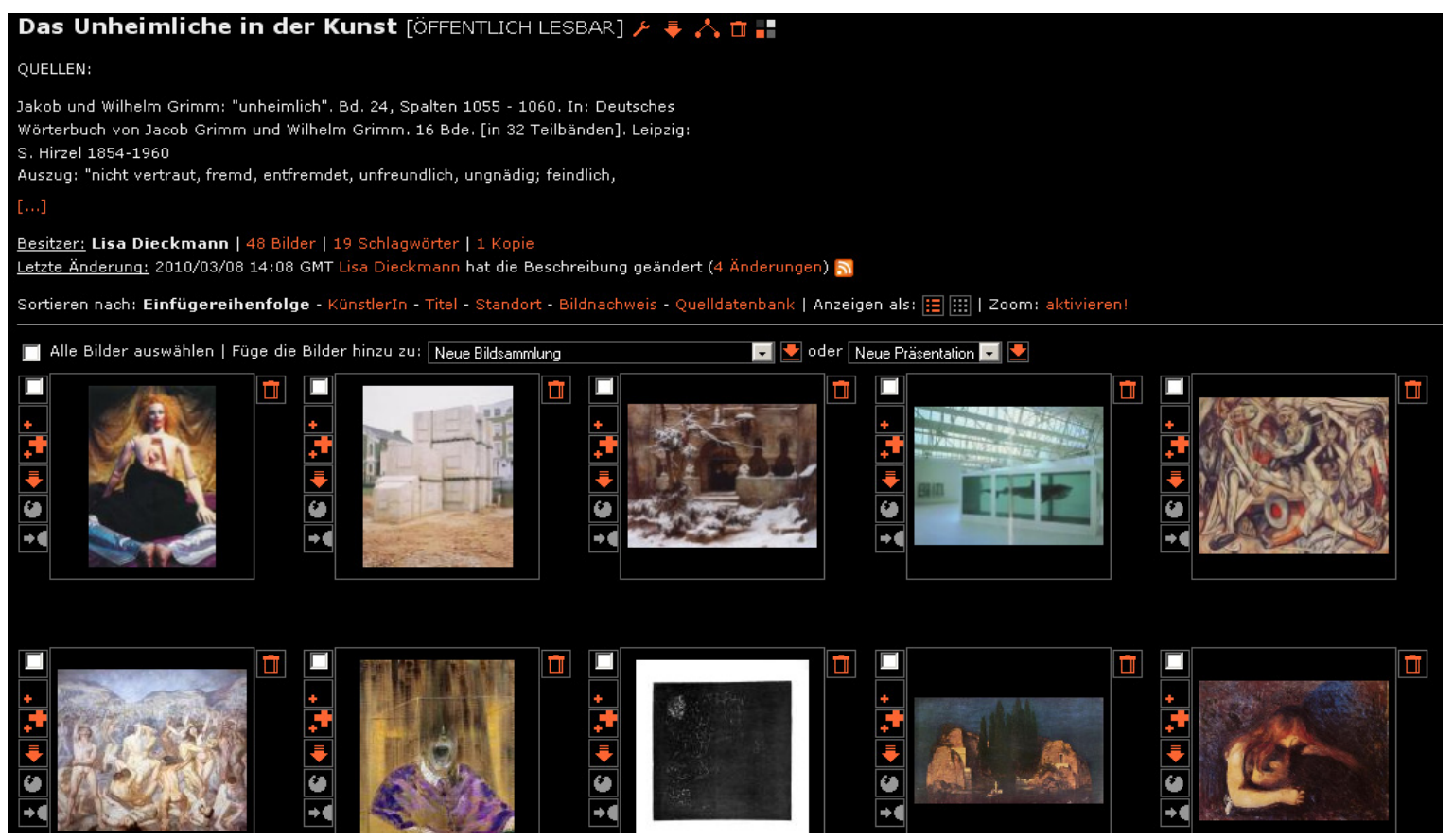

Fig. 5 - Publicly readable prometheus-collection

Summing up these features: prometheus consists of a very large pool of images and connected image databases, as well as the facility for collaborative working, a large number of users and a legally secure background. It therefore offers the perfect foundation for creating new orders of knowledge, new insights, and research questions for imagebased research. 


\section{HYPERIMAGE - AN IMAGE ANNOTATION TOOL}

HyperImage is a tool for image-annotation, which allows the identification of motifs, the creation of image networks and links, and the addition of metadata to images. The combination of prometheus and HyperImage offers a powerful instrument to support art historical or other visualculture studies.

HyperImage has been developed by a project funded by the German Federal Ministry of Education and Research from 2006 to 2009 at Leuphana University Lüneburg in collaboration with the Computer and Media Service at Humboldt University Berlin. It enables the user to work with images, as Aby Warburg did, offering precise annotation, the linking and indexing of images and their details in a way that until now was only possible for text-based hyperlinks. HyperImage consists of the HyperImage Editor and the HyperImage Reader. The HyperImage Editor allows images to be edited in a variety of ways - to upload and classify images, edit metadata, create and edit explanatory notes, add layers on images to highlight details and to hyperlink whole images or just specific elements.

The HyperImage Reader conveys a viewing mode for the work results of the Editor.

\subsection{WORKING WITH THE HYPERIMAGE EDITOR}

The following example will show how indexing structures can be created with the Editor in order to be used for presentation in the Reader. Carmen Wedemeyer, Martin Warnke and Christian Terstegge from Leuphana University Lueneburg used HyperImage for an approach to the work of the Hamburg-based artist Anna Oppermann (19401993). Oppermann created so-called Ensembles, consisting of up to thousands of drawings, photographs, canvases, sculptures, tablets and scrolls. These elements are interrelated through a complex cross-referential image-in-image structure, which can be made visible through the connections made in HyperImage. The example shows the indexing of the Ensemble Besinnungsobjekte über das Thema Verehrung - Anlass Goethe (Objects of Reflection on the Subject of Adoration - On the Occasion of Goethe).

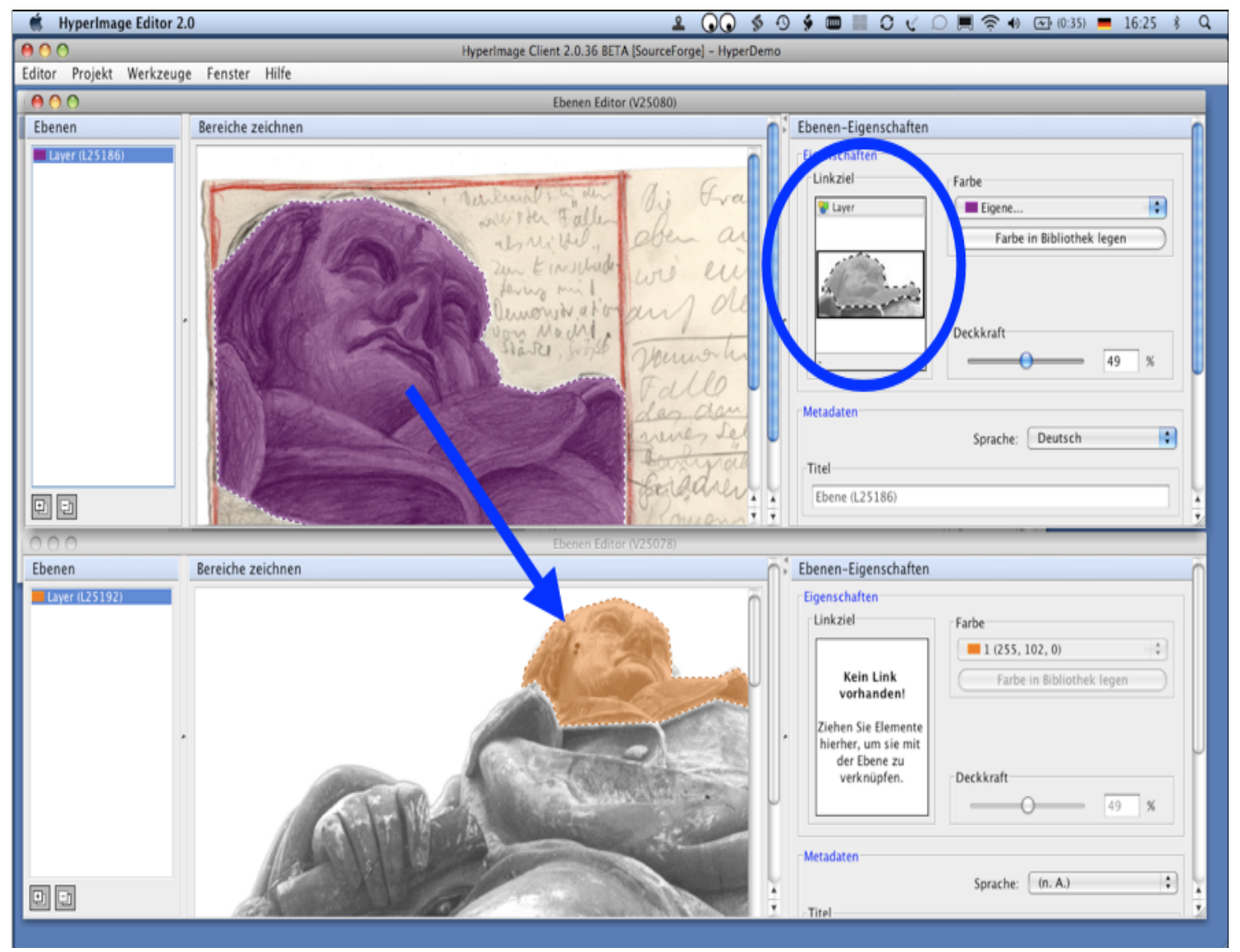

Fig. 6 - The Linkage of two details in the Editor. Here the upper head is linked to the other head below, which appears in the blue circle as the link's target 


\subsection{THE HYPERIMAGE READER AND ITS FUNCTIONS}

ensemble or they can retrace the process of its

In reading mode the user can follow the links from the total view to a detailed view of the

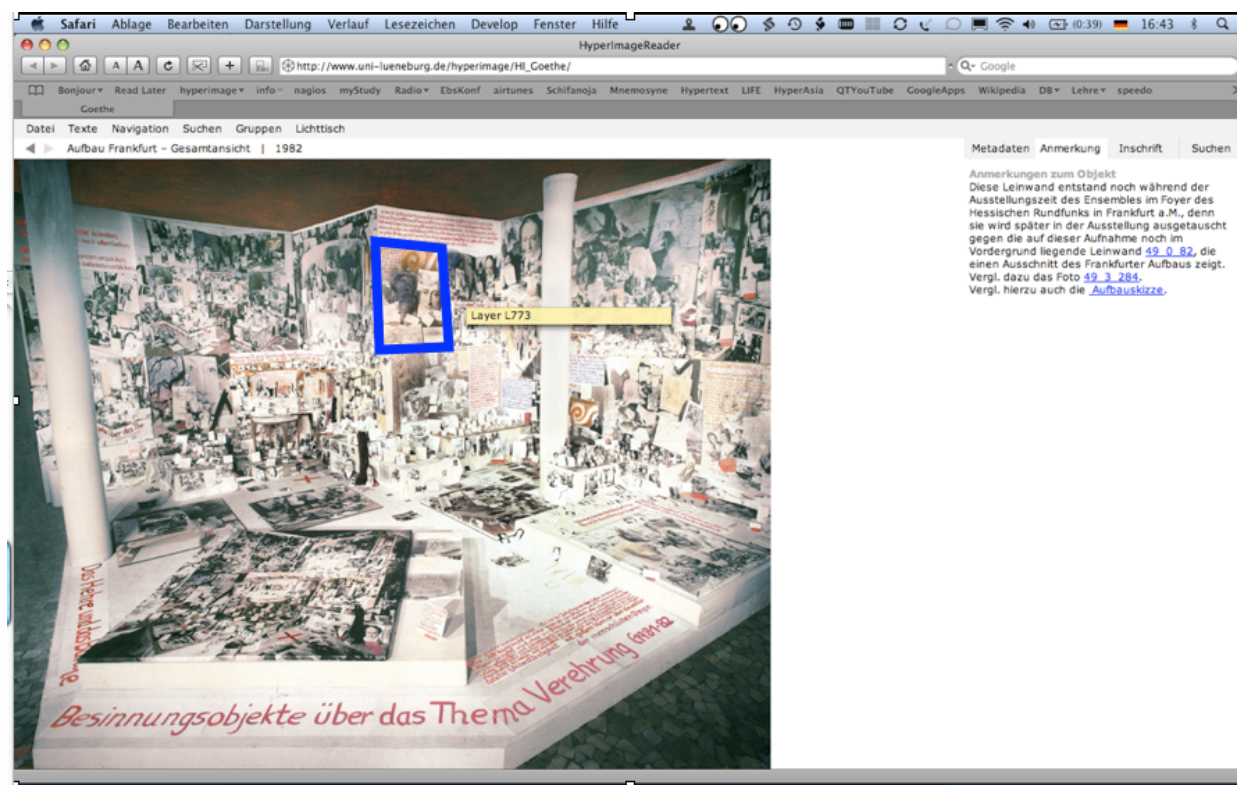

Fig. 7 - Total view of the Ensemble in 1982 in Frankfurt am Main (here as in the following figures a blue frame highlights the relevant details)

Clicking on the highlighted areas of the image elements.

takes the user into a detailed view of single

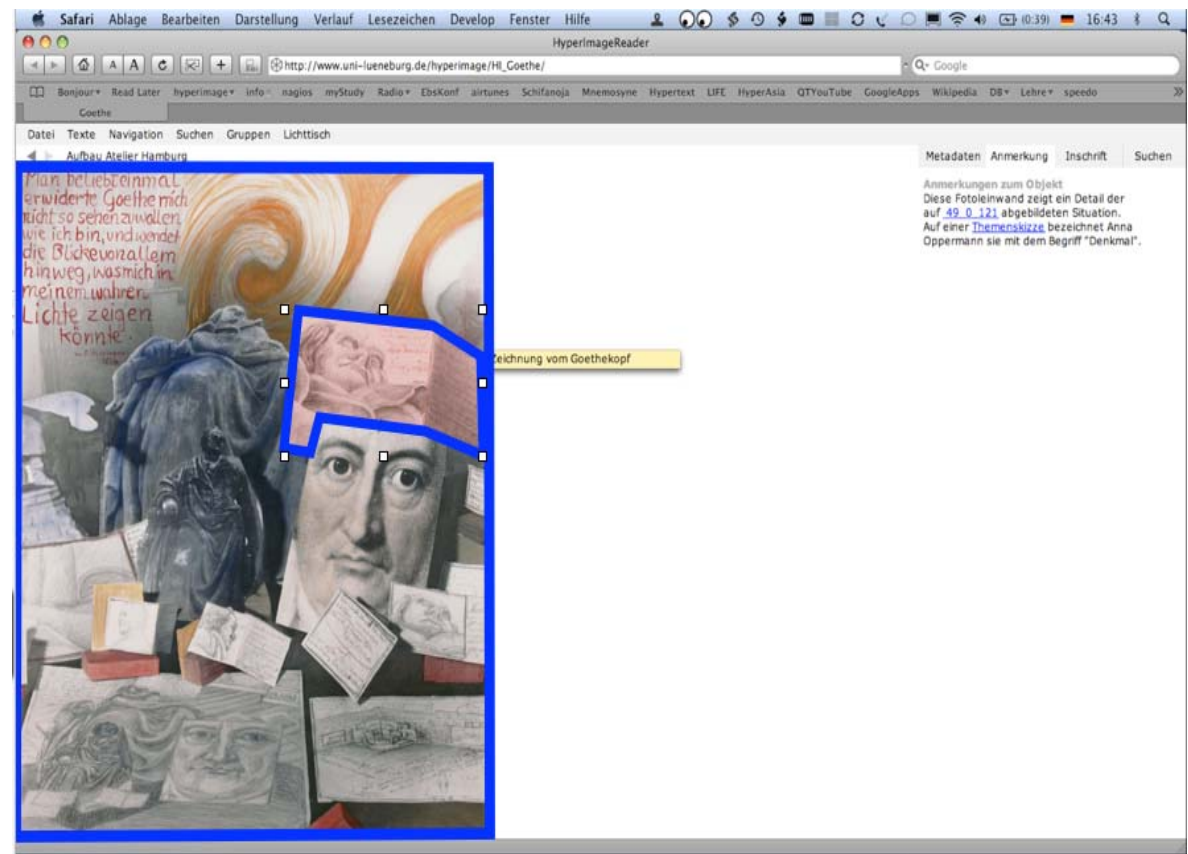

Fig. 8 - Detailed view of a single element of the previous image, with the next detail (drawing) highlighted

Next to the image, metadata and explanatory notes are displayed, which further describe the object as a photo canvas. The highlighted detail of this image is a drawing of Goethe's head, which can also be clicked on. 


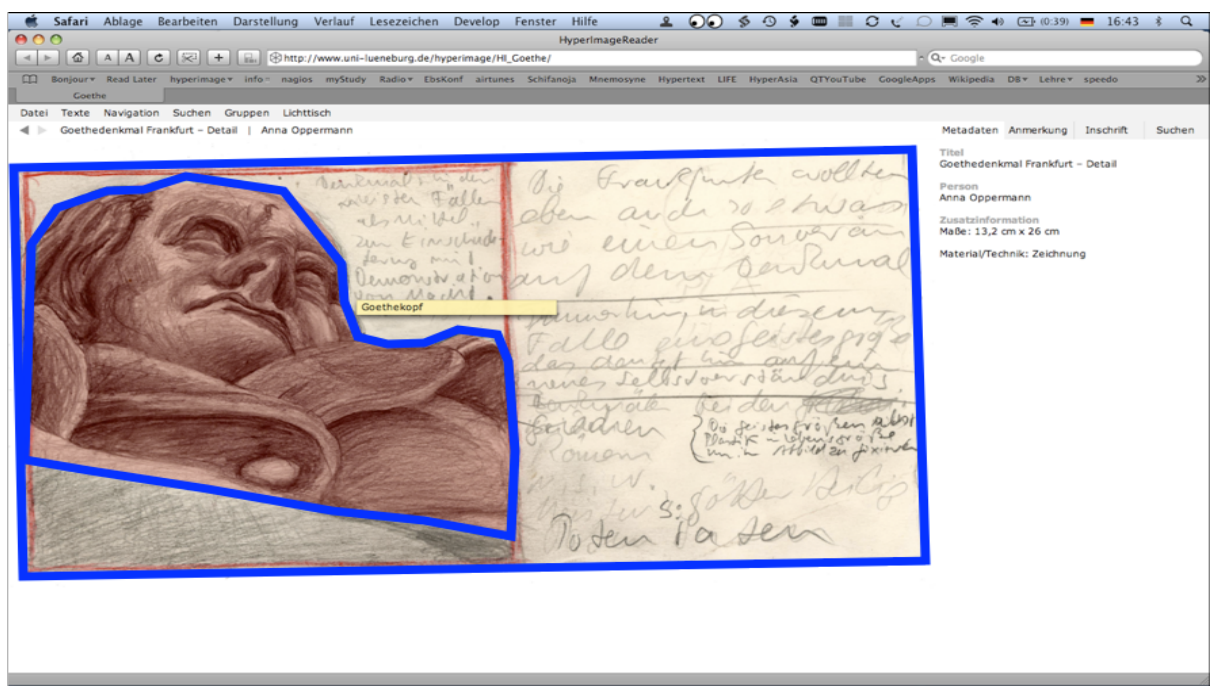

Fig. 9 - Detail view of the Goethe drawing with the head highlighted

Clicking on the drawing of the head leads to a Main, which served as a template for the drawing detailed image of the Goethe statue in Frankfurt am and before was referenced within the Editor.

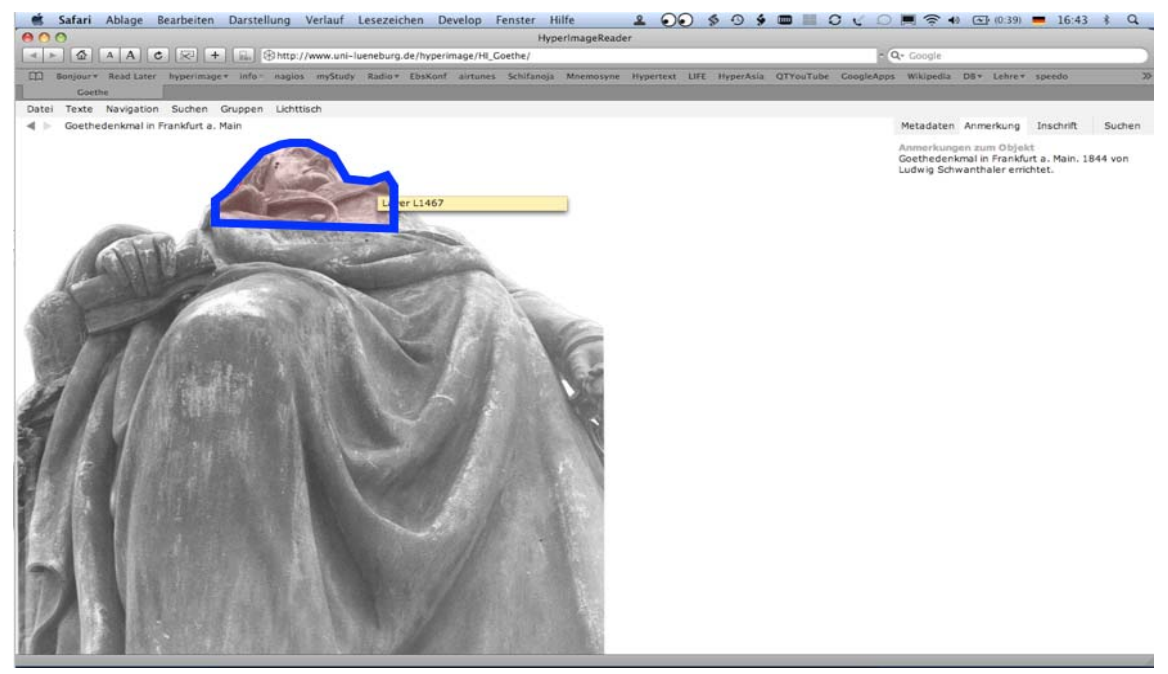

Fig. 10 - Photography of the Goethe statue in Frankfurt am Main

HyperImage supports collaborative research on images by means of two further functions. The light table view makes it possible to scale objects and arrange them in different ways in order to compare them directly. The image index function shows all the references, from the displayed image to other objects, as well as all the references going back to the displayed image. The image index corresponds with an index or a concordance in text culture. For collaborative research on images the image index is particularly useful in opening up new contexts of interpretation for an image or its motif.

This way of representing relationships between images is based on the Mnemosyne Atlas by Aby Warburg. In a similar way, he juxtaposed and compared images with each other in order to demonstrate coherences and similarities among them [4].

Besides the connecting structure, which results from linking the various image details, there are explanatory notes and metadata serving as advanced cross-references. Thus users are able to explore the work following their own areas of interest and are not bound to a fixed linking structure or to prearranged interpretations.

As seen in the example of Anna Oppermann's work, the referencing system of HyperImage is able to structure and index image corpora. Visual connections become traceable, images can be set in direct comparison to each other or the material can be arranged into a visual argument. HyperImage facilitates - in the sense of Warburg - reasoning with the help of images, which leads to a visual network for collaborative research on images as explained above.

\section{META-IMAGE - THE CONNECTION OF PROMETHEUS AND HYPERIMAGE}

Meta-Image is based on prometheus and works 
with the tool HyperImage. It forms the infrastructure which enables the user to work on image details individually or in a group. To realise this project, both technologies had to be augmented by special functions and made interoperable in a user-friendly way.

The main functions remain unaffected, and technical modularity is achieved by defining interfaces conforming to standards. For the exchange of data, prometheus provides a web service which is used by the Meta-Image Editor.

When clicking on the Meta-Image symbol within the prometheus collection the images of the respective collection are transferred into the MetaImage Editor. The metadata of the images will also be passed to the editor. The full functionality, however, is only provided for users with the appropriate permission.

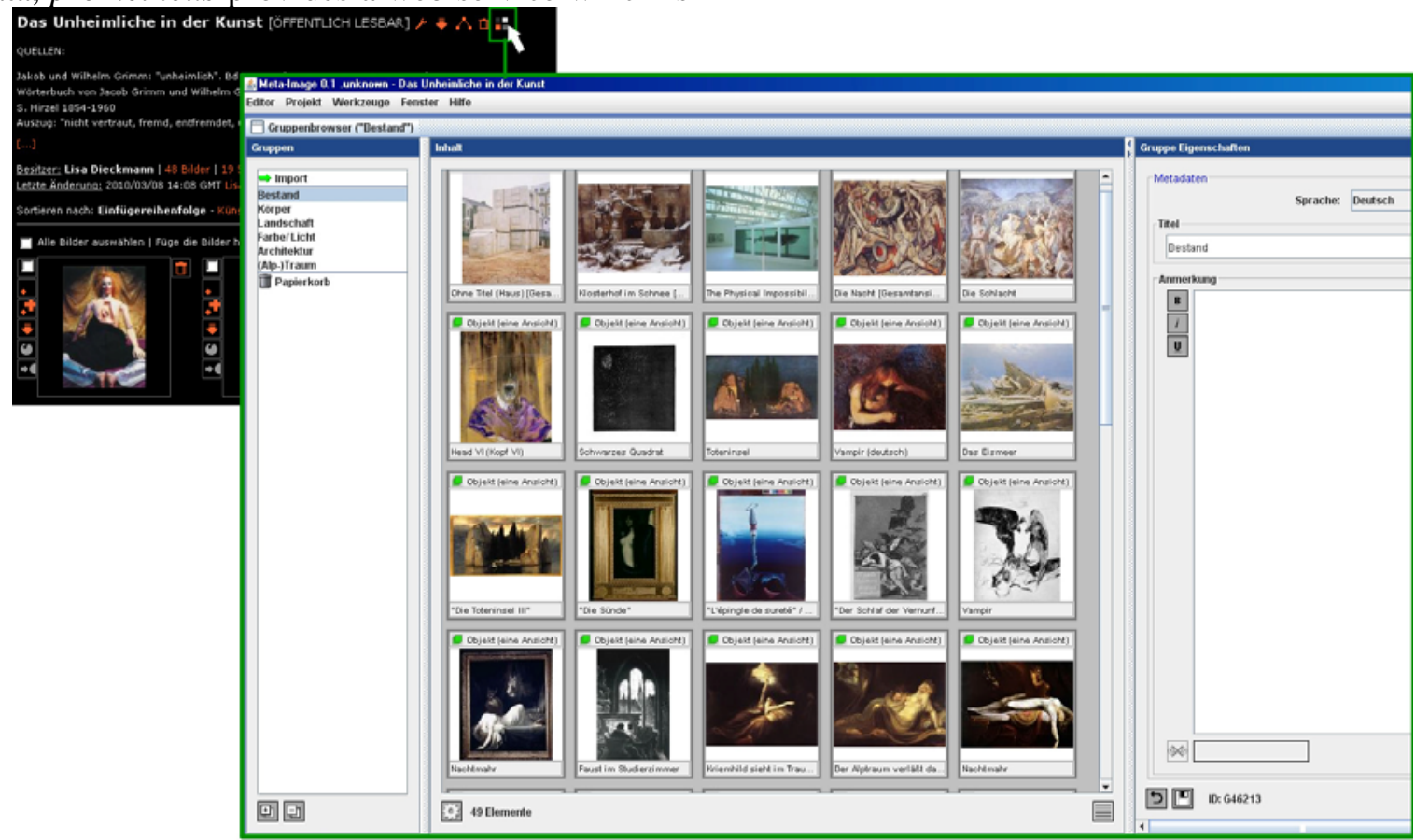

Fig. 11. - Consuming the web service by clicking the Meta-Image symbol and transferred the data into the MetaImage-Editor

The Meta-Image Editor is only accessible via prometheus, which also handles access rights. Authorisation for editing a collection is defined there and cannot be changed in Meta-Image. Users, who are editing the collection in the Meta-Image Editor, are permitted to do nearly everything they want to: add metadata, create layers, mark image details and create links between layers or images. But they are not permitted to upload images into the Meta-Imagecollection. This is to ensure the consistency of the collection in Meta-Image and prometheus. This means that the user has to upload the images into their own personal prometheus collection. This function is not implemented yet but will be soon.

The formal documentary metadata of the images in prometheus will be enhanced by the results of the informal analysis in Meta-Image: Meta-image results will be written to prometheus. Source and context always remain transparent and connected to the relevant author. This process does not affect the original metadata of the records, which are provided in the integrated databases. Whether this information can be used for image retrieval will be discussed during the course of the project.
Once the results of the research in the MetaImage-Editor have been published, a link to the Meta-Image Reader will appear in the corresponding prometheus collection.

Summing up, the various functions and aspects of prometheus and HyperImage ensure that the relevant research material is made available and resources are pooled as connected databases. The network-based infrastructure and secure legal access to the material for research and education are location independent. Both technologies offer interactive and collaborative structures to analyse and display image structures and connections directly on the images.

Combining these functions and advantages, Meta-Image delivers optimal conditions for network-based and collaborative image discourse in art history and visual culture studies.

In his trilogy The Rise of the Network Society Manuel Castells discovers that 'the technology does not determine society, but embodies it' [5]. The media are offering new and different horizons of possibilities and spaces for activity, but society has to decide whether to choose stability or destruction [6]. We are now at the point of decision: whether to 
benefit from the possibilities of hypertext applied to media, and interactivity of the medium for the image discourse or not. We really think we should embrace this opportunity!

\section{ACKNOWLEDGEMENT}

Supported by the German Research Foundation (DFG) under the project title 'Aufbau einer verteilten virtuellen Forschungs- und Kommunikationsumgebung für den Bilddiskurs in der Kunstgeschichte (Meta-Image), [Development of a distributed virtual research and communication environment for the image discourse in art history (Meta-Image)], DI 1574/1-1, SCHI 491/7-1, WA 2404/1-1, 2009-2011, in the program Scientific Library Services and Information Systems (LIS).

\section{REFERENCES}

[1] Boehm G. Die Wiederkehr der Bilder. In Boehm, G. (ed.) Was ist ein Bild? Wilhelm Fink Verlag, München, 1994.

[2] Rorty R. The Linguistic Turn. Chicago, 1967.

[3] Mitchell W. J. T. Iconology - Image, Text, Ideology. Chicago, 1986.

[4] Huisstede P. V. Der Mnemosyne-Atlas. Ein Laboratorium der Bildgeschichte, 1955.

[5] Castells M. Der Aufstieg der Netzwerkgesellschaft. Teil 1 der Trilogie. Das Informationszeitalter. Opladen, 2001.

[6] Simon H. Kunstgeschichte im digitalen Informationszeitalter - Eine kritische Standortbestimmung (Plenumsvortrag am 14. März 07 auf dem 29. Deutschen Kunsthistorikertag in Regensburg). In Art-Dok. Publikationsplattform Kunstgeschichte, Heidelberg, 2007, p. 3.

\section{NOTES ON FIGURES}

Figures 1 and 2

http://commons.wikimedia.org/wiki/File:

Robert_Campin_012.jpg (2 August 2010)

Figures 3 to 5

Screenshots of http://www.prometheus-bildarchiv.de (29 March 2010)

Figures 6 to 10

Screenshots taken from the HyperImage project

'Anlass Goethe' (On the Occasion of Goethe)

http://www.uni-lueneburg.de/hyperimage/

HI_Goethe/ (29 March 2010)

Figure 11

Screenshots from the Meta-Image pilot project on the development server at University of Cologne

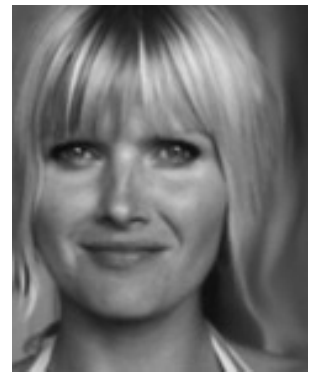

Lisa Dieckmann studied Art History, Humanities Computer Science and German Literature in Cologne and Rome and graduated in 2004 with a Master's degree. Since 2005 she has been working as research assistant and managing director for the project "prometheus - the distributed digital image archive for research and education" (see http://www.prometheusbildarchiv.de) at the Institute of Art History at the University of Cologne. From 2007-2009 she also worked at the research institute "Lichtbildarchiv älterer Originalurkunden" for the correspondent research project at the University of Marburg and coordinated the realization of the online platform. She has successfully worked for several research projects, such as currently "Meta-Image" (http://www.meta-image.de). She is also a founding member of the Cologne Center for eHumanties at the University of Cologne. After the collapse of the Cologne Stadtarchiv (city archive) in 2009 she was part of the research group who initiated the "Digital Historical Archive Cologne" (see http://www.historischesarchivkoeln.de). As a doctoral candidate in Art History she is writing her dissertation on "Dream Imagery in German Romanticism". Her particular scientific interests are in particular: 19th century Art, digital art, digital humanities and new media.

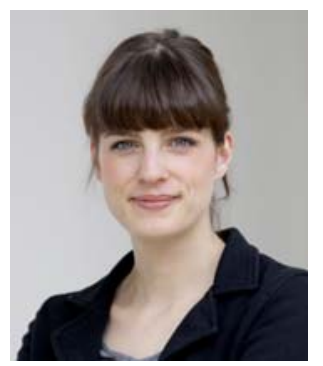

Anita Kliemann studied Cultural Sciences and Media Studies in Lüneburg and graduated in 2009 with a Master's Degree. Her main interests are visual culture, media art, new media and film. She currently is working as a research assistant at Leuphana University of Lüneburg for the project "Meta-Image A Collaborative Environment for Image Discourse in Art History" (see http://www.meta-image.de) funded by the German Research Foundation (DFG). She is responsible for public relations, user support and software documentation.

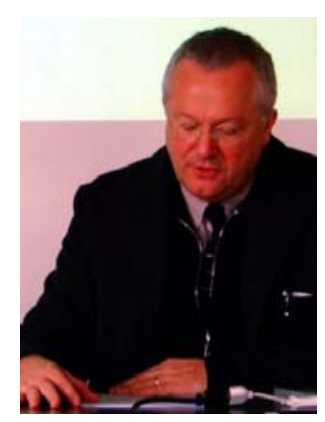

PD Dr. Martin Warnke studied in Berlin and Hamburg, graduated in Hamburg and is now Associate Professor for Computer Science and Digital Media in Lüneburg. As the one who initiated the department Computers and Culture he developed the curriculum that combines cultural, artistic and computer science issues theoretically and practically. Since 1983 he taught contiously in Lüneburg, Basel, Klagenfurt, and 
Vienna. In several projects together with artists and students he developed and produced artistic works on digital media. He successfully worked on a number of research projects: See www.metaimage.de and www.hyperimage.eu Together with collegues from Lüneburg, Berlin and Basel he established the annual "HyperKult"-Workshops and the discourse that evolved with it. He is engaged in the German Computer Society ("Gesellschaft für Informatik e. V.), has been chair of the working group "Computers as Media" and until 2008 chair of the technical committee "Computers and Society" of GI e. V. Since April 2009 he is the chairperson of the Kunstverein Springhornhof (art club in Neuenkirchen). 